University of New Hampshire

University of New Hampshire Scholars' Repository

Space Science Center

Institute for the Study of Earth, Oceans, and

Space (EOS)

2005

\title{
Atmospheric neutron measurements with the SONTRAC science model
}

U Bravar

University of New Hampshire - Main Campus

E O. Fluckiger

University of Bern

Kevin Godin

University of New Hampshire - Main Campus

Zachary C. Hansen

University of New Hampshire - Main Campus

John R. Macri

University of New Hampshire - Main Campus, John.Macri@unh.edu

See next page for additional authors

Follow this and additional works at: https://scholars.unh.edu/ssc

Part of the Astrophysics and Astronomy Commons

\section{Recommended Citation}

Bravar, U.; Fluckiger, E.O.; Godin, K.; Hansen, Z.C.; Macri, J.R.; McConnell, M.L.; Miller, R.S.; Moser, M.R.; Ryan, J.M., "Atmospheric neutron measurements with the SONTRAC science model," Nuclear Science Symposium Conference Record, 2005 IEEE , vol.2, no., pp.634,638, 23-29 Oct. 2005

This Conference Proceeding is brought to you for free and open access by the Institute for the Study of Earth, Oceans, and Space (EOS) at University of New Hampshire Scholars' Repository. It has been accepted for inclusion in Space Science Center by an authorized administrator of University of New Hampshire Scholars' Repository. For more information, please contact Scholarly.Communication@unh.edu. 
Authors

U Bravar, E O. Fluckiger, Kevin Godin, Zachary C. Hansen, John R. Macri, Mark L. McConnell, R S. Miller, M R. Moser, and James M. Ryan 


\title{
Atmospheric Neutron Measurements with the SONTRAC Science Model
}

\author{
Ulisse Bravar, Erwin O. Flückiger, Kevin Godin, Zachary C. Hansen, John R. Macri, Mark L. McConnell, Richard S. \\ Miller, Michael R. Moser, and James M. Ryan
}

\begin{abstract}
The SOlar Neutron TRACking (SONTRAC) telescope was originally developed to measure the energy spectrum and incident direction of neutrons produced in solar flares, in the energy range $20-250 \mathrm{MeV}$. While developed primarily for solar physics, the SONTRAC detector may be employed in virtually any application requiring both energy measurement and imaging capabilities. The SONTRAC Science Model (SM) is presently being operated at the University of New Hampshire (UNH) as a ground-based instrument to investigate the energy spectrum, zenith and azimuth angle dependence of the cosmic-ray induced sea-level atmospheric neutron flux. SONTRAC measurements are based on the non-relativistic double scatter of neutrons off ambient protons within a block of scintillating fibers. Using the n-p elastic double-scatter technique, it is possible to uniquely determine the neutron's energy and direction on an event-by-event basis. The 3D SM consists of a cube of orthogonal plastic scintillating fiber layers with $5 \mathrm{~cm}$ sides, read out by two CCD cameras. Two orthogonal imaging chains allow full 3D reconstruction of scattered proton tracks.
\end{abstract}

\section{AtMosPheric NEUTRONS}

A TMOSPHERIC neutrons are produced by galactic and solar cosmic rays as they hit nitrogen and oxygen nuclei in the Earth's atmosphere and generate both hadronic and electromagnetic air showers.

Secondary neutrons in the 10-200 MeV energy range are among the most problematic of cosmic-ray particles. In recent years, these neutrons have become a growing concern in the context of avionics reliability. The ${ }^{28} \mathrm{Si}\left(\mathrm{n}, \mathrm{n}^{\prime} \alpha\right){ }^{24} \mathrm{Mg}$ reaction releases an energetic, heavily ionizing $\alpha$ particle. The ionization can change the state of a solid-state junction, producing a single rvent upset (SEU), i.e. a nondestructive

Manuscript received November 11, 2005. This work was supported in part by NASA under grant NNG04GM61G in the United States and the Swiss National Science Foundation under grant 200020-105435/1 in Switzerland.

$\mathrm{U}$. Bravar is with the Space Science Center, University of New Hampshire, Durham, NH 03824, USA (e-mail: ulisse.bravar@unh.edu).

E. O. Flückiger is with the Physikalisches Institut, University of Bern, CH-3012 Bern, Switzerland (e-mail: erwin.flueckiger@phim.unibe.ch).

K. Godin is with the Space Science Center, University of New Hampshire, Durham, NH 03824, USA (e-mail: godin@cisunix.unh.edu).

Z. C. Hansen is with the Space Science Center, University of New Hampshire, Durham, NH 03824, USA (e-mail: zhansen@cisunix.unh.edu).

J. R. Macri is with the Space Science Center, University of New Hampshire, Durham, NH 03824, USA (e-mail: john.macri@unh.edu).

M. L. McConnell is with the Space Science Center, University of New Hampshire, Durham, NH 03824, USA (e-mail: mark.mcconnell@unh.edu).

R. S. Miller is with the Department of Physics, University of Alabama, Huntsville, AL 35899, USA (e-mail: millerr@email.uah.edu).

M. R. Moser is with the Physikalisches Institut, University of Bern, CH-3012 Bern, Switzerland (e-mail: michael.moser@phim.unibe.ch).

J. M. Ryan is with the Space Science Center, University of New Hampshire, Durham, NH 03824, USA (e-mail: james.ryan@unh.edu). circuit failure. With the increasing density of modern circuitry, the potential for radiation effects increases. Highdensity circuits are the most sensitive to soft errors. As a consequence, new circuitry must be designed with a greater fraction of error-correction channels, thereby diminishing the potential minification of electronic chips [1], [2].

Ground level upsets have also been observed in computer systems with large allocations of random access memory (RAM). Furthering our knowledge of atmospheric neutrons, the major cause of this type of upsets, would allow us to:

1. improve the reliability of large computer systems;

2. implement error mitigation techniques for RAMs in biomedical, industrial and commercial products;

3. develop realistic microelectronics reliability standards.

Probably of greater importance is the effect of neutrons on flight crews at aircraft altitudes and in space. The general behavior of the downward-moving neutron flux is that it grows linearly from the top of the atmosphere downward, reaching a maximum at an atmospheric depth of $100 \mathrm{~g} / \mathrm{cm}^{2}$, known as the Pfotzer Maximum [3]. This peak corresponds to an altitude of approximately $20 \mathrm{~km}$. At lower altitudes, the downward-moving neutron flux decreases exponentially until sea level. The upward moving albedo flux also decreases exponentially from the Pfotzer Maximum toward sea level, but above the maximum the upward-moving flux is relatively constant extending outward to low Earth orbit. At the Pfotzer Maximum, the flux is roughly isotropic.

Finally, sea-level atmospheric neutrons represent one of the main backgrounds in the detection of Special Nuclear Material (SNM) through passive or active neutron interrogation of cargo containers. Unlike other forms of radiation produced by $\mathrm{SNM}$, copious and penetrating neutron emission in the $\mathrm{MeV}$ energy range is unique to fissionable material. Consequently, development of SNM detection techniques based on neutron imaging is one of the highest priority $R \& D$ areas in the application of nuclear science to homeland security. To effectively design instrumentation to scan for illicit amounts of SNM, the flux of background neutrons at the search site must be known accurately, including the shape and any major features in the energy spectrum, as well as the zenith and azimuth angle dependences [4].

\section{SEA-LEVEl NeUtron MEASUREMENTS}

In spite of their relevance, few measurements of the energy spectrum of atmospheric neutrons in the $\mathrm{MeV}$ energy range are available in literature. Over 100 neutron monitors are in continuous operation throughout the world, including the 
University of New Hampshire (UNH) monitors located on Mt. Washington, $\mathrm{NH}$ (the longest running monitor in the world since 1954) and on the UNH main campus in Durham, NH [5]. However, these instruments typically function only as neutron counters, measuring time variations in the integral neutron flux, with no sensitivity to the neutron's energy and to the zenith and azimuth angles of its track.

A compilation of the energy spectra measured from the 1950s through the 1990s can be found in [6], while most recent measurements were published by [7] and [8]. The spectrum reported by [8] is shown in Fig. 1, with flux values obtained from data measured by different detectors within a factor of two of each other.

Uncertainties in the zenith angle dependence are significant. According to [9], the sea-level neutron flux varies as $\cos ^{\mathrm{n}} \theta$, with $\mathrm{n}=3.5 \pm 1.2$, up to a zenith angle $\theta=60^{\circ}$. The azimuthal asymmetry is expected to be small, but has not been determined to this day. A more precise measurement that includes a detailed analysis of the zenith and azimuth dependence of the sea-level neutron flux would be beneficial especially in modeling the background for SNM detectors.

\section{SONTRAC}

The SOlar Neutron TRACking imaging spectrometer (SONTRAC), described in detail in the following sections, was first developed to measure the energy spectrum and incident direction of neutrons produced in solar flares, in the energy range $20-250 \mathrm{MeV}$, from high-altitude balloon or low-Earth orbit satellite experiments [10]. The measurement of "background" atmospheric neutrons, both downwardmoving and upward-moving albedo, was also one of the initial secondary science goals. The intense neutron albedo in lowEarth orbit requires that imaging techniques be employed to maximize an instrument's signal-to-noise ratio.

While developed primarily for solar physics, the SONTRAC detector may be employed in virtually any application requiring both energy measurement and imaging capabilities. It will appear clear in the following sections that the event-byevent accuracy of SONTRAC is unsurpassed by past and present detectors. Considering that a fully operational 3D science model (SM) of SONTRAC had been manufactured and performance testing had been completed (see Fig. 2), we chose to operate this detector in conjunction with the neutron monitors on the UNH main campus to study the sea-level atmospheric neutron flux. We expect our measurements to complement and improve the spectral data presently available in literature and provide a benchmark measurement of the zenith and azimuth dependences of the neutron flux.

The SONTRAC instrument measures the interaction position, track direction and kinetic energy of recoil protons from n-p elastic scatters in plastic scintillator. Central to this instrument is a bundle composed of mutually perpendicular, alternating layers of parallel scintillating plastic fibers. Viewed by opto-electronic devices the bundle provides two

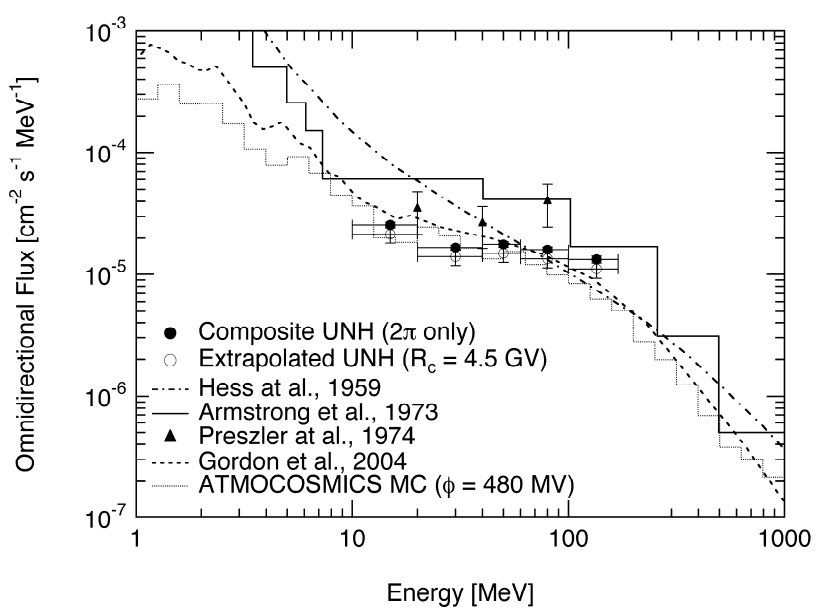

Fig. 1. Measured energy spectrum of the sea-level atmospheric neutron flux. Courtesy of [8].

orthogonal views of recoil proton tracks. The resulting stereoscopic data are used to determine the incident neutron's direction and energy on an event-by-event basis, as explained in the following sections.

\section{NEUTRON DETECTION}

The only demonstrated technique to image a neutron source at $\mathrm{MeV}$ energies is by measuring recoil protons from elastic $\mathrm{n}$ p scatters [11]. Neutrons themselves have no electric charge and therefore cannot be detected directly. Simple nonrelativistic kinematic considerations show that it is advantageous to have neutrons scatter off hydrogen (i.e. protons) rather than larger-Z nuclei. Organic scintillators offer the advantage of being both an ideal scatterer and detector material.

Figure 3 illustrates the topology of the double-scatter of a non-relativistic neutron in a solid block of plastic scintillator. Neutrons predominantly interact in plastic either by elastically scattering from hydrogen (n-p) or by interacting with carbon $(n-C)$. As said, the $n-p$ events are the most useful. For nonrelativistic scattering:

$$
\sin ^{2} \theta_{\mathrm{n}}=\frac{E_{p 1}}{E_{n}}
$$

where $E_{n}$ is the incident neutron's energy, $E_{p l}$ is the scattered proton's energy and $\theta_{n}$ is the recoil neutron's scatter angle. The scattered neutron and proton momenta lie at right angles to one another.

In the case of an event where only a single recoil proton is detected, providing that the vector momentum of this proton can be determined, some limited information on the incident neutron can be extracted on an event-by-event basis, namely:

1. if the incident direction of a given neutron is known, $\theta_{n}$ is uniquely determined and is sufficient to reconstruct the incident neutron's energy; 


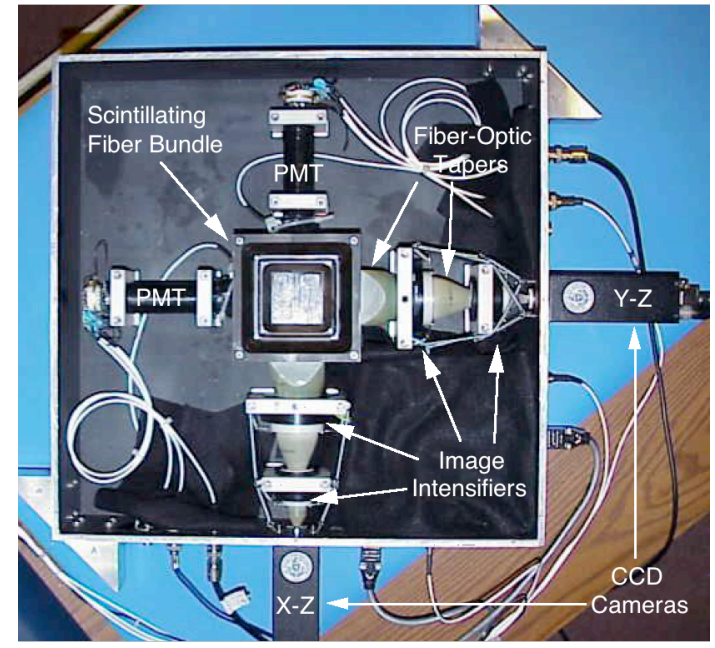

Fig. 2. The SONTRAC 3D SM, seen from above. The plastic scintillator fiber bundle is visible in the center of this image. To the top and left are the two PMTs used for trigger. To the right and bottom are the two optic chains with image intensifiers, coupled to two CCD cameras.

2. if the incident neutron's energy is known, its direction can be constrained as lying on the mantle of a cone from a single $n-p$ scatter.

However, single n-p scatters do not allow for the determination of both the neutron's incident angle and energy simultaneously.

A more deterministic approach is provided by double-scatter events, such as the case displayed in Fig. 3. If both the energy and direction of the two recoil protons are measured, then the energy and incident direction of the neutron are uniquely determined. A system capable of measuring the parameters of both recoil proton tracks in three dimensions therefore provides the necessary information to unambiguously determine the incident neutron's parameters.

The technique described here differs from the method used in the $2-20 \mathrm{MeV}$ range by the Fast Neutron Imaging Telescope (FNIT) [12]. In FNIT, recoil proton energies are too low to produce a detectable track and one can measure the interaction vertex and proton's kinetic energy only.

\section{THE SONTRAC 3D SM}

After successfully demonstrating the basic detection concept with a small 2D prototype, a full 3D Science Model of the SONTRAC instrument was manufactured. The SONTRAC 3D SM was performance tested at a particle beam in 2001 [10]. Its response characteristics were determined in full detail and the instrument is now completely operational.

The 3D SM consists of a tracking detector cube $5 \mathrm{~cm} \times 5 \mathrm{~cm}$ $\times 5 \mathrm{~cm}$ in size that includes over 27,000 plastic scintillating fibers in both horizontal coordinates, complemented by appropriately sized optoelectronic readout components. The scintillating fibers serve both as the source of ambient proton scattering centers, and as light pipes for image readout. A photographic image of this cube is shown in Fig. 4. The square plastic fibers are arranged in stacked planes with the

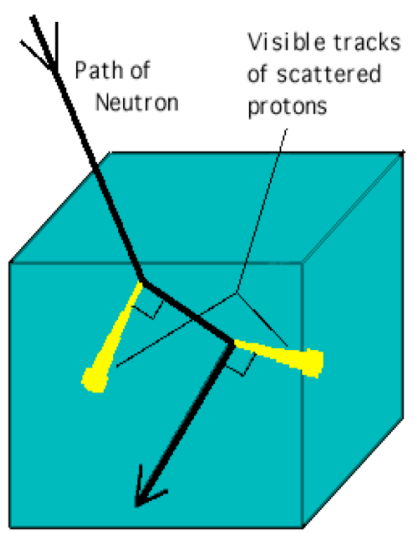

Fig. 3. Double elastic n-p scatter in a plastic scintillator cube. The tracks of the two recoils from each interaction form a right angle. By measuring the energies and directions of the two recoil protons (shown in yellow gold), one can reconstruct the direction and energy of the incident neutron.

fibers in each plane orthogonal to those of overlying and underlying planes. This alternating orientation of fiber planes provides a stereoscopic view of recoil proton tracks and allows for the reconstruction of these tracks in three dimensions. Ionizing proton tracks are then used to determine the arrival direction and energy of incident neutrons. The resulting neutron angular and energy resolutions depend upon the precision with which the recoil proton direction and energy can be determined.

The horizontal and vertical fiber pitch in the SONTRAC $\mathrm{SM}$ is $300 \mu \mathrm{m}$. BCF-99-55MC plastic fibers are used [13], with the size of the active core approximately $250 \mu \mathrm{m}$. The experimental setup was already shown in Fig. 2. The fiber bundle is seen by two orthogonal photomultiplier tubes (PMTs) and by two orthogonal optic chains that include image tapers, image intensifiers and CCD cameras. The plastic scintillating fiber detector bundle, as well as the light guides, fiber optic tapers, image intensifiers, and associated power supplies are contained within a single light-tight enclosure (cover removed in Fig. 2). The two orthogonal CCD cameras are mounted externally. In this setup, the SONTRAC SM has no ancillary detector elements such as anti-coincidence or calorimeter. External equipment, not shown in Fig. 2, includes NIM electronics and a CAMAC ADC for processing the PMT signals and performing the trigger logic, and the computer for control and data acquisition.

The SM is self-triggered. A twofold coincidence of the PMTs provides the trigger for gating on the image intensifiers and capturing the two orthogonal track images with the CCD cameras. The intensity of each pixel's recorded scintillation light combined with the inherent pixelization of the fiber bundle provides the information for track reconstruction. The origin and signal intensity along the track are used to discriminate the type of radiation detected (i.e. proton, neutron, muon...). For particles that range out within the fiber bundle, the ionization track length provides a good measure of the particle energy and the Bragg peak, corresponding to the 


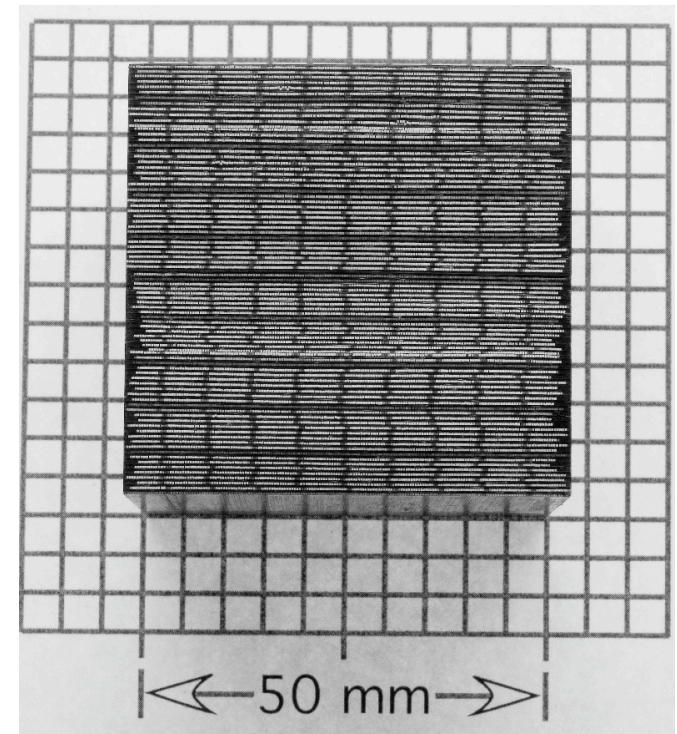

Fig. 4. Photographic image of one of the side surfaces of the SONTRAC SM plastic scintillator fiber cube. Individual fibers are clearly visible.

greater ionization near the end of a particle track, identifies the particle direction.

The size of the SM fiber bundle permits measurements to be made from 20 to $150 \mathrm{MeV}$, a range starting at the nominal threshold up to energies where neutron double scatter events are no longer contained. The energy $E_{p}$ of the recoil proton in the SONTRAC SM can be parametrized as a function of its track length $d_{p}$ as:

$$
\mathrm{E}_{\mathrm{p}}=\mathrm{E}_{0} \times\left(\mathrm{d}_{\mathrm{p}} / \mathrm{d}_{0}\right)^{\alpha}
$$

where the parameters $E_{0}, d_{0}, \alpha$ were obtained by Monte Carlo simulations and checked against beam data $\left(\mathrm{E}_{0}=35.2 \mathrm{MeV}\right.$, $\mathrm{d}_{0}=1 \mathrm{~cm}, \alpha=0.546$ ). Taking into account that fiber pitch is $300 \mu \mathrm{m}$, a recoil proton must have a kinetic energy of at least $8 \mathrm{MeV}$ in order for its range in the SM to be at least two fibers. Therefore, the low-energy limit of the SM for neutrons, when operated in the double-scatter mode, is $\sim 20$ $\mathrm{MeV}$. The high-energy limit is fixed by double-scatter event containment. In principle, it could be raised by manufacturing a larger scintillating fiber bundle. However, the recoil proton track length increases almost quadratically with the energy and the inelastic n-p cross-section becomes significant at energies above $400 \mathrm{MeV}$, raising above the elastic one. When this happens, the double n-p scatter technique can no longer be employed and a different type of detector, e.g. a calorimeter, has to be used.

The energy and angular resolutions of the SONTRAC SM for recoil protons have been determined to be $\delta \mathrm{E} / \mathrm{E}=4.8 \%$ and $\delta \theta=4.6^{\circ}$ at $35 \mathrm{MeV}$, improving with energy [10]. The SM prototype was calibrated with proton beams and it was demonstrated that heavily ionizing protons are easily recognized and measured. Future possible developments of this instrument include lower channel-count opto-electronic readout devices and improved algorithms for processing and analyzing data.

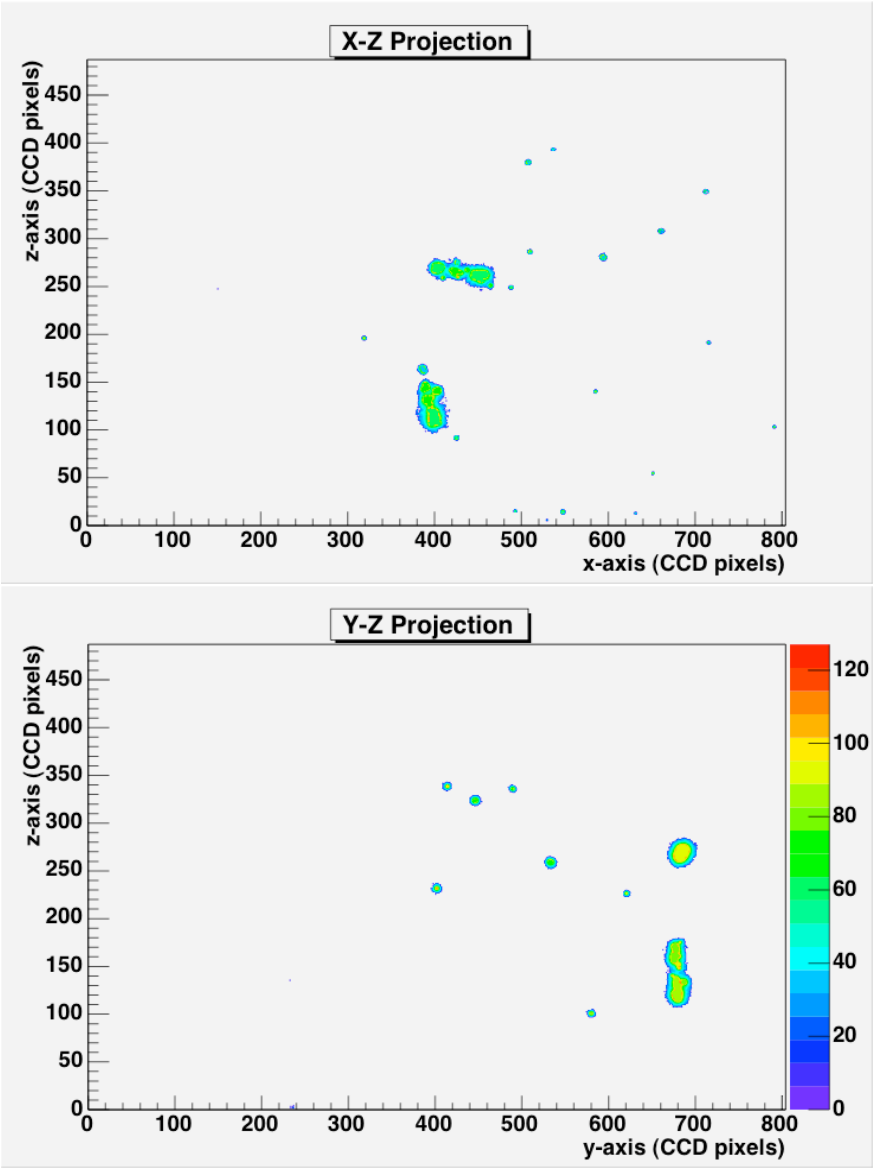

Fig. 5. A double-scatter event produced by an atmospheric neutron as seen by the SONTRAC SM detector. The primary neutron was traveling downwards along the z-axis. The tracks of the two recoil protons are clearly visible in both SONTRAC projections onto the $(\mathrm{x}-\mathrm{z})$ and $(\mathrm{y}-\mathrm{z})$ planes (top and bottom, respectively). From this image, the energy and direction of the incident neutron can be reconstructed.

\section{AtMospheric NeUtron MEASUREMENTS With SONTRAC}

From the above description, it appears clear that SONTRAC has the capability of providing an extremely accurate measurement of the sea-level atmospheric neutron flux, by far exceeding the precision of present-day detectors. Considering the relevance of the neutron background at aircraft altitudes and the small size and self-contained design of SONTRAC, a future application may also include the use of this detector on commercial airliners. The only major drawback of SONTRAC when used for atmospheric neutron measurements is represented by its small size, requiring relatively long exposure times.

In September 2005 we set up the SONTRAC 3D SM in the hall housing the UNH neutron monitors located on the main campus and started acquiring atmospheric neutron data. The lack of any significant amount of material in the roof structure on top on SONTRAC guarantees that we are measuring the unbiased atmospheric neutron flux. The energy threshold of the two PMTs had to be kept relatively low in order for theSM 
to trigger on $8 \mathrm{MeV}$ protons. Since the SM was self-triggered, this low threshold caused miminum ionizing cosmic-ray muons to produce a trigger signal as well. Therefore, we chose to install a plastic scintillator layer with a thickness of $0.5 \mathrm{~cm}$ on top of the SONTRAC SM to serve as an anticoincidence shield.

With this setup, the SONTRAC SM was operated continuously during the month of October 2005. We achieved a trigger rate of $0.02 \mathrm{~Hz}$. Of the acquired events, only about $1.6 \%$ were identified as single-scatter or double-scatter neutrons. During an effective exposure time of $\sim 30$ days, we counted 574 single n-p scatters and 9 double n-p scatters. Figure 5 shows one of the detected double-scatter events. The two proton tracks are clearly visible. Table I summarizes our observed data.

As part of the SM development, a fully functional Monte Carlo simulation code, based on the GEANT4 package [14], was developed as well. We are presently in the process of analyzing our atmospheric neutron data to validate the Monte Carlo predictions. So far, we used the known atmospheric neutron flux as the input for our Monte Carlo runs and checked the simulated detector response against our experimental data. A valuable crosscheck tool is represented by the double-scatter to single-scatter event ratio. Its dependence on the input spectrum used in the Monte Carlo is weak at best. In addition to crosscheck for Monte Carlo and/or data analysis algorithm tuning, this quantity provides a direct measurement of the SONTRAC SM detector efficiency. Results achieved so far are satisfactory but not perfect, with the experimental and Monte Carlo values of this ratio consistently within the same order of magnitude, prompting us to improve our software and analysis techniques even further.

After we achieve an acceptable level of agreement between experimental and Monte Carlo data, we plan on using the simulation code to disentangle the atmospheric neutron flux from single n-p scatter events. In principle, the measured distributions of track length, zenith and azimuth angles of single-scatters in the SONTRAC SM should be reflected in the Monte Carlo data, providing the atmospheric neutron energy spectrum and angular distribution used as the input to the simulation code are representative of the actual sea-level flux. Due to the paucity of double-scatter events, the analysis of single-scatter data combined with Monte Carlo simulations has the potential of becoming the ultimate tool for our atmospheric measurements. As an added bonus, the energy threshold of the SM for single-scatters is $\sim 10 \mathrm{MeV}$.
TABLE I

SingLE- AND DOUBLE-SCATTER EVENTS IN EXPERIMENTAL DATA

\begin{tabular}{cccc} 
& $\begin{array}{c}\text { single- } \\
\text { scatters }\end{array}$ & $\begin{array}{c}\text { double- } \\
\text { scatters }\end{array}$ & $\begin{array}{c}\text { double / } \\
\text { single ratio }\end{array}$ \\
\hline Exp. Data & 574 & 9 & $1.6 \%$ \\
\hline
\end{tabular}

\section{CONCLUSIONS}

We described the structure and performance of the SONTRAC 3D SM. This instrument is presently being operated as a sea-level atmospheric neutron detector. At the time of writing, it has been in continuous operation for a period of over one month. The first measurements are encouraging, although we do not yet have sufficient statistics for a full quantitative analysis. We plan to keep collecting atmospheric neutron data with the SONTRAC SM for the foreseeable future.

\section{REFERENCES}

[1] J. F. Ziegler, and W. A. Lanford, "Effect of Cosmic Rays on Computer Memories," Science, vol. 206, pp. 776-788, 1979.

[2] H. H. K. Tang, and K. P. Rodbell, " Single-event upsets in microelectronics," MRS Bulletin, vol. 28, pp. 117-120, 2003.

[3] A. Preszler, et al., J. Geophys. Res., vol. 79, p. 17, 1974.

[4] L. Forman, P. E. Vanier, and K. E. Welsh, "Distinguishing spontaneous fission neutrons from cosmic-ray background," Proc. SPIE, vol. 5541, pp. 27-36, 2004.

[5] http://wwwgro.sr.unh.edu/neutron_monitors/

[6] P. K. F. Grieder, Cosmic rays at Earth: researcher's reference manual and data book, Amsterdam: Elsevier, 2001, pp. 335-341.

[7] M. S. Gordon, et al., "Measurement of the Flux and Energy Spectrum of Cosmic-Ray Induced Neutrons on the Ground," Proc. IEEE Nucl. Sp. Rad. Effects Conf., 2004.

[8] M. R. Moser, J. M. Ryan, L. Desorgher, and E. O. Flückiger, "Atmospheric Neutron Measurements in the 10-170 MeV Range," Proc. $29^{\text {th }}$ Int. Cosmic Ray Conf., SH35, pp. 101-104, 2005.

[9] E. Heidbreder, K. Pinkau, C. Reppin, and V. Schönfelder, J. Geophys. Res., vol. 76, p. 2905, 1971.

[10] R.S. Miller, J.R. Macri, M.L. McConnell, J.M. Ryan, E. Flückiger, and L. Desorgher, "SONTRAC: An imaging spectrometer for $\mathrm{MeV}$ neutrons," Nucl. Inst. Meth., vol. A505, pp. 36-40, 2003.

[11] H. Debrunner, et al., "Neutrons fron the 15 June 1991 Solar Flare," Proc. $23^{\text {rd }}$ Int. Cosmic Ray Conf., vol. 3, pp. 115-118, 1993.

[12] U. Bravar, et al., "Development of the Fast Neutron Imaging Telescope," these proceedings.

[13] http://www.photonic.saint-gobain.com

[14] S. Agostinelli, et al., "GEANT4: A Simulation Toolkit," Nucl. Inst. Meth., vol. A506, pp. 250-303, 2003. 